\title{
Vancomycin-resistant enterococci (VRE) in Canada - Results of the Canadian Nosocomial Infection Surveillance Program 1996 VRE point prevalence surveillance project
}

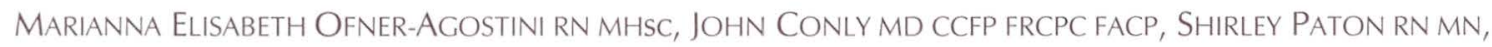

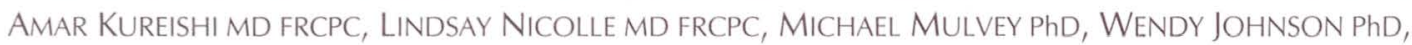 \\ LYNN JOHNSTON MD FRCPC AND THE CANADIAN HOSPITAL EPIDEMIOLOGY COMMITTEE
}

$\mathrm{E}$ nterococci are the second most commonly isolated nosocomial pathogen and the third most commonly isolated pathogen associated with nosocomial bacteremias (1-3). The National Nosocomial Infection Surveillance (NNIS) system in the United States reported a 20 -fold increase in the percentage of nosocomial enterococcal isolates that were vancomycin-resistant between 1989 and 1993 (2). Outbreaks of vancomycinresistant enterococci (VRE) have been reported throughout the United States, occurring in both acute and long term care facilities (4-6). Laboratory-based statewide surveillance in the United States has been implemented for VRE in New Jersey and Connecticut $(7,8)$, in nine of 33 states with NNIS hospitals in the United States (9) and in 44 hospitals in the United Kingdom (10). While individual hospitals in Canada have collected information on the occurrence and effects of VRE, there have been no aggregate Canadian data collected to date.

The published literature primarily reflects hospital-specific

Division of Nosocomial and Occupational infections, Bureau of Infectious Diseases, Laboratory Centre for Disease Control, Ottawa, Ontario

Correspondence and reprints: Dr Marianna Ofner-Agnosti, Division of Nosocomial and Occupational Infections, Bureau of Infectious Diseases, Laboratory Centre for Disease Control, Third Floor, LCDC Building, Tunney's Pasture, Ottawa, Ontario K1A OL2. Telephone 416-978-0373, fax 416-978-8299, e-mail m.ofner@utoronto.ca
VRE prevalence studies or laboratory-based isolate surveillance. Patients with VRE have been identified in renal dialysis units, nursing homes, infant-toddler surgical wards, pediatric oncology units, medical and surgical units, neonatal intensive care units (ICUs), ICUs and hemo-oncology wards (4,5,11-14). These patients are considered high risk patients. The occurrence of VRE has since been identified in hospitalized nonhigh risk groups in Canada (unpublished data) and in the United States $(4,15)$.

Infections caused by VRE can be difficult to treat because these organisms are inherently resistant to most antimicrobials. Of additional concern is the ability of enterococcus to transfer antimicrobial resistance traits to other organisms. In vitro and in vivo research have demonstrated conjugative transfer of high-level vancomycin resistance from Enterococcus faecalis to Staphylococcus aureus (16). Although gene transfer has not been identified in a human host, the threat exists. Therefore, surveillance for and control of this organism are considered important.

To determine the prevalence of VRE within hospitals across Canada, the Canadian Nosocomial Infection Surveillance Program (CNISP), a collaborative effort of the Canadian Hospital Epidemiology Committee (CHEC), a subcommittee of the Canadian Infectious Diseases Society and the Laboratory Centre for Disease Control (LCDC), Health Canada, implemented a VRE point prevalence surveillance project within selected Canadian hospitals. The purpose of the project was to collect epi- 
demiological and laboratory information to predict valid estimations of the magnitude and impact of VRE in high risk patients in Canada.

\section{BACKGROUND}

A preproject survey was sent to CHEC members to determine the history of VRE occurrences in their associated hospitals, the surveillance programs in place and the control measures implemented (17). In the last quarter of 1995, 21 CHEC members, working in both pediatric and adult tertiary care teaching hospitals, completed the preproject survey. Fiftyfive per cent of the sites had performed surveillance for VRE within their institution. Significant variations in methods used, and patient groups surveyed were described by each of these institutions. In total, 2690 specimens had been tested for VRE. Four sites identified 34 patients with VRE (vancomycin minimal inhibitory concentration levels ranging from 8 to $128 \mu \mathrm{g} / \mathrm{mL}$ ). Thirty-one patients were colonized with VRE, and three were infected. Nine sites $(43 \%)$ reported restricting the use of vancomycin, although three sites only restricted the use of oral vancomycin. The average amount of vancomycin dispensed by the hospital pharmacies per patient-year from 1991 to 1994 had significantly increased over time $(r=0.94)$. The main conclusions drawn from the preproject survey were that VRE had arrived in Canada; variable screening was occurring with respect to types of specimens, patient groups and frequency of testing; and the use of vancomycin was increasing significantly. As a result of these findings, the CNISP 1996 VRE point prevalence surveillance project was developed and implemented in January 1996. The results of this project are presented in this paper.

\section{PATIENTS AND METHODOLOGY}

CHEC facilities and their affiliated hospitals were invited to participate in the point prevalence surveillance project if the facility had one of the following units: a surgical ICU, medical ICU, combined medical-surgical ICU, neonatal ICU, hematology-oncology unit, bone marrow transplant unit, solid organ transplant unit and hemo-peritoneal dialysis unit. Patients receiving care on these units were considered high risk patients. All in-patients who received care in these units were eligible to be enrolled in the study, provided they had been hospitalized on that ward or bed for at least four days. Outpatients receiving dialysis in these hospitals were also eligible for enrolment. Sites collected data for a continuous four weeks sometime between January 1996 and March 1996.

The facility information collected included the number of ward and ICU admissions and the average number of filled beds over the four-week period. For the purposes of this survey, an endemic hospital was defined as one in which an outbreak of VRE had been identified in the past year. An endemic patient group was defined as a group or ward of patients who experienced an outbreak within the past year.

Information collected from each participating patient included date of birth, sex, bed type, date of admission, laboratory specimen collection date, present use of antibiotics and vancomycin and routes of vancomycin, if used. The length of stay was defined as the time between date of admission and laboratory specimen collection date. Present vancomycin use was collected and reported as 'yes' if the patient was receiving it at the time the specimen was collected or, for patients with renal failure, if it had been given within seven days before the date that the specimen was obtained. A stool or rectal swab was obtained from eligible patients for VRE testing.

An additional detailed questionnaire was administered to all patients found to have VRE. Information recorded on the detailed questionnaire included diagnosis, underlying chronic diseases, recent surgery, elimination pattern, presence of Clostridium difficile toxin, activity level and recent nonsurgical invasive procedures. Detailed questionnaires were not completed by the VRE-negative patients.

VRE-positive patients were compared with VRE-negative patients using $\chi^{2}$ or Fisher exact tests for categorical values. Continuous variable comparisons were performed using the Student's $t$ test, Wilcoxon rank-sum test or the Kruskal-Wallis $\mathrm{H}$ test.

\section{LABORATORY MATERIALS AND METHODS}

Bacterial isolates: Screening of stool and rectal swab specimens for VRE used m-enterococcus agar containing $6 \mu \mathrm{g} / \mathrm{mL}$ vancomycin (Difco, Michigan). Presumptive glycopeptide-resistant enterococci were submitted to LCDC. After confirmation of vancomycin resistance on brain-heart infusion agar containing $6 \mu \mathrm{g} / \mathrm{mL}$ vancomycin (18), isolates were identified using standard laboratory procedures. E faecium and Enterococcus faecalis isolates were then coded and distributed to participating laboratories for further study.

Antimicrobial susceptibility testing: All isolates were tested by agar dilution as described by the National Committee for Clinical Laboratory Standards (19). Antimicrobial agents tested were vancomycin, teicoplanin, ampicillin, penicillin, gentamicin, streptomycin, tetracycline, doxycycline and chloramphenicol.

Genetic analysis: Detection of van A and van B genes by polymerase chain reaction was performed as described by DutkaMalen et al (20). Pulsed-field gel electrophoresis (PFGE) was conducted using the restriction enzyme Smal by the method of Murray et al (21). Banding patterns were interpreted according to published criteria (22).

\section{RESULTS}

Twenty-six Canadian hospitals participated in the CNISP 1996 VRE point prevalence surveillance project. Participating hospitals included 12 hospitals from Ontario $(51.8 \%$ of the total isolates submitted), three from Alberta (16.5\%), two from Manitoba $(9.2 \%)$, four from Quebec $(8.9 \%)$, two from Nova Scotia $(5.2 \%)$, one from Saskatchewan $(3.3 \%)$, one from New Brunswick $(2.9 \%)$ and one from Newfoundland $(2.2 \%)$. One hospital in Ontario was classified as an endemic hospital because an outbreak of VRE had occurred among hemodialysis patients in 1995

A total of 3773 patients were enrolled. VRE was isolated from 26 patients, one from Alberta and 25 from Ontario. Fourteen (53.8\%) of the VRE-positive patients were in-patients 
TABLE 1

Source of isolates, antibiotic resistance profiles and molecular typing

\begin{tabular}{|c|c|c|c|c|c|}
\hline Identifier & Province & Species & PCR genotype & Resistance antibiogram* & PFGE pattern \\
\hline N96-126 & Ontario & EFE & B & VA, AP, PN, GM, SM & A1 \\
\hline N96-127 & Ontario & EFE & B & VA, AP, PN, GM, SM & A7 \\
\hline N96-129 & Ontario & EFE & B & VA, AP, PN, GM, SM, TC, DC & B \\
\hline N96-130 & Ontario & EFE & B & VA, AP, PN, GM, SM & $\mathrm{A} 1$ \\
\hline N96-132 & Ontario & EFE & B & VA, AP, PN, GM, SM & A5 \\
\hline N96-134 & Ontario & EFE & B & $\mathrm{VA}, \mathrm{AP}, \mathrm{PN}, \mathrm{SM}$ & A6 \\
\hline N96-135 & Ontario & EFE & B & VA, AP, PN, SM, TC & $\mathrm{C} 1$ \\
\hline N96-136 & Ontario & EFE & B & VA, AP, PN, SM & $\mathrm{C} 2$ \\
\hline N96-137 & Ontario & EFE & B & VA, AP, PN, GM, SM & A1 \\
\hline N96-138 & Ontario & EFE & B & $\mathrm{VA}, \mathrm{AP}, \mathrm{PN}$ & A1 \\
\hline N96-143 & Ontario & EFE & B & VA, AP, PN, SM & A4 \\
\hline N96-144 & Ontario & EFE & B & $\mathrm{VA}, \mathrm{AP}, \mathrm{PN}, \mathrm{SM}$ & $\mathrm{A} 2$ \\
\hline N96-145 & Ontario & EFE & B & VA, AP, PN, GM, SM, TC, DC & A3 \\
\hline N96-146 & Ontario & EFE & B & VA, AP, PN, SM & $\mathrm{A} 2$ \\
\hline N96-147 & Ontario & EFE & B & VA, AP, PN SM & A8 \\
\hline N96-148 & Ontario & EFE & B & VA, AP, PN, GM, SM & $\mathrm{A} 2$ \\
\hline N96-149 & Ontario & EFE & B & VA, AP, PN, GM, SM & A1 \\
\hline N96-159 & Ontario & EFE & $\mathrm{B}$ & VA, AP, PN, GM, SM & A1 \\
\hline N96-125 & Ontario & EFC & B & VA, GM, TC & $\mathrm{D}$ \\
\hline N96-131 & Ontario & EFC & B & VA, GM, TC, DC & $\mathrm{D}$ \\
\hline
\end{tabular}

*Minimal inhibitatory concentration breakpoints were as follows: vancomycin (VA) $\leq 4 \mu \mathrm{g} / \mathrm{mL}$ for susceptible, 8 to $16 \mu \mathrm{g} / \mathrm{mL}$ for intermediate and $\geq 32 \mu \mathrm{g} / \mathrm{mL}$ for resistant; teicoplanin (TP) $\leq 8 \mu \mathrm{g} / \mathrm{mL}$ for susceptible, $16 \mu \mathrm{g} / \mathrm{mL}$ for intermediate and $\geq 32 \mu \mathrm{g} / \mathrm{mL}$ for resistant; ampicillin (AP) $\leq 8 \mu \mathrm{g} / \mathrm{mL}$ for susceptible and $\geq 16 \mu \mathrm{g} / \mathrm{mL}$ for resistant; penicillin (PN) $\leq 8 \mu \mathrm{g} / \mathrm{mL}$ for susceptible and $\geq 16 \mu \mathrm{g} / \mathrm{mL}$ for resistant; high level gentamicin (GM) $>500 \mu \mathrm{g} / \mathrm{mL}$ for resistant; high level streptomycin (SM) > 2000 $\mu \mathrm{g} / \mathrm{mL}$ for resistant; tetracycline (TC) and doxycycline (DC) $\leq 4 \mu \mathrm{g} / \mathrm{mL}$ for susceptible, $8 \mu \mathrm{g} / \mathrm{mL}$ for intermediate and $\geq 16 \mu \mathrm{g} / \mathrm{mL}$ for resistant; and chloramphenicol (CL) $\leq 8 \mu \mathrm{g} / \mathrm{mL}$ for susceptible, $16 \mu \mathrm{g} / \mathrm{mL}$ for intermediate and $\geq 32 \mu \mathrm{g} / \mathrm{mL}$ for resistant. A Polymerase chain reaction (PCR) genotype vanA gene; B PCR genotype vanB gene; EFE Enterococcus faecium; EFC Enterococcus faecalis PFGE Pulsed-field gel electrophoresis

and $12(46.2 \%)$ were out-patients. The endemic hospital reported 23 VRE-positive patients; two other VRE-positive patients received treatment at this hospital. The prevalence rate was one per $1000(0.1 \%)$ high risk patients in a hospital with no outbreaks (nonendemic), 37 per 1000 (3.7\%) high risk patients in the endemic hospital, and 53 per 1000 (5.3\%) high risk patients in the endemic patient group (hemodialysis) in the endemic hospital.

Of the 26 VRE isolates, three were E faecalis (11.5\%) and 23 were $E$ faecium $(88.5 \%)$. Source of isolates, antibiotic resistance profiles and molecular typing are presented in Table 1. All E faecium isolates were resistant to ampicillin and penicillin, whereas none of the $E$ faecalis isolates demonstrated resistance to the beta-lactams. All but one isolate showed high level resistance to aminoglycosides. Approximately $25 \%$ of the strains exhibited resistance to either tetracycline and doxycycline, whereas no chloramphenicol-resistant strains were identified. More than $96 \%$ (25 of 26 ) of the isolates contained the $\operatorname{van} \mathrm{B}$ gene that also corresponded to the geographical area of isolate collection.

The majority of patients surveyed were from the dialysis, hemo-oncology and medical-surgical ICU wards/beds (Figure 1). Most of the enrolled patients were dialysis patients. Of the VRE-positive patients, 92\% (24 patients) were receiving dialysis. The other two VRE cases were in hemo-oncology and medical ICU wards. No VRE cases were identified in the surgical, medical-surgical or neonatal ICUs or on solid organ transplant units. Patients receiving dialysis had a 9.2 times greater chance of being VRE-positive than if they belonged to any other patient category (OR 9.2; 95\% CI 3.05, 38.49; P<0.001). A patient receiving dialysis in Toronto was 20 times $(95 \% \mathrm{CI}$ $7.33,67.38 ; \mathrm{P}<0.001)$ more likely to be VRE-positive than any other study participant, and those receiving dialysis in Toronto were 24.7 times ( $95 \%$ CI 4.59, 514.68; P<0.001) more likely to be VRE-positive compared with the rest of the dialysis population. 


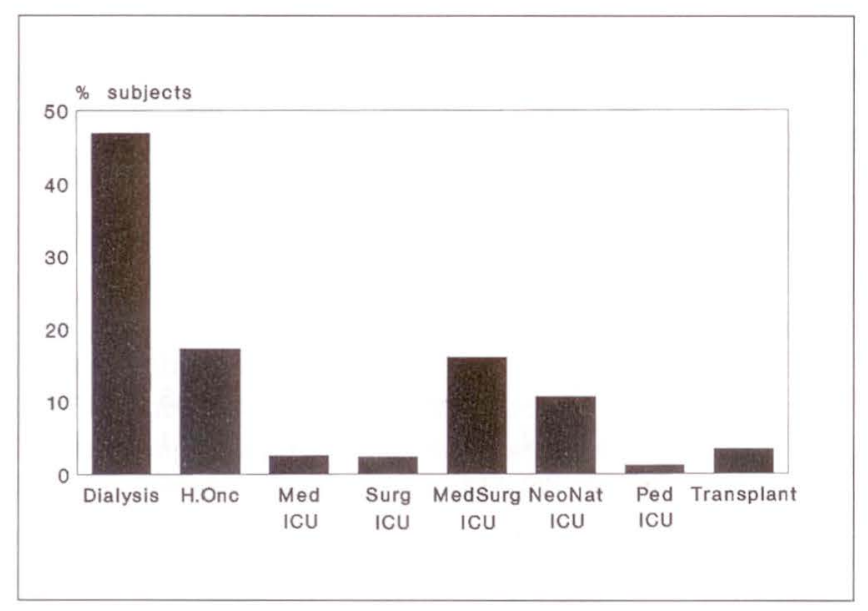

Figure 1) Bed type categories: percentage participants in vancomycin-resistant enterococci point prevalence surveillance. H.Onc Hemo-oncology; ICU Intensive care unit; Med Medical; NeoNat Neonatal; Ped Pediatric; Surg Surgical; Transplant Solid organ transplant

Sex was similar for VRE-negative $(56.6 \%$ male and $43.3 \%$ female) and VRE-positive patients $(56.7 \%$ and $43.4 \%$, respectively). Mean age of VRE-positive patients was 59 years, and mean age of VRE-negative patients was 52 years, a nonsignificant difference. None of the VRE-positive patients was less than 20 years of age.

There were no significant differences between VRE-positive and -negative patients with respect to antibiotic use. For the VRE-negative patients, $31 \%$ were receiving antibiotics at the time of laboratory specimen collection (51.3\% of these were on one antibiotic, $34.2 \%$ were on two antibiotics and $14.5 \%$ were on three or more); $34 \%$ of VRE-positive patients were receiving antibiotics $(55.5 \%$ one antibiotic, $33.3 \%$ two antibiotics and $11.1 \%$ three or more). The average time each VRE-positive patient had been on an antibiotic before specimen collection was 10.5 days (range two to 25). This information was unavailable for the VRE-negative patients.

At the time of laboratory specimen collection, 1169 patients (31\% of the total population surveyed) were receiving antibiotics, $13 \%(152)$ of these were receiving vancomycin. None of the VRE-positive patients were receiving vancomycin. The route of administration was intravenous in $134(88 \%)$, oral in four $(2.6 \%)$, intramuscular in two (1.3\%) and intraperitoneal in two (1.3\%).

VRE-positive in-patients had a significantly $(\mathrm{P}<0.01)$ longer length of hospital stay than VRE-negative in-patients (Figure 2). The average length of stay for the VRE-negative in-patients was 19 days (median seven days; mode four days). The average length of stay for VRE-positive in-patients was 58 days (median 21 days; mode 14 days).

VRE-positive patients - detailed questionnaire results: Admitting diagnoses for the $14 \mathrm{VRE}$ in-patients included acute renal failure, peritonitis, cardiomyopathy, $C$ difficile-associated diarrhea infection, dehydration and a gangrenous foot. Fourteen (52\%) VRE patients were ambulatory, five (18.5\%) had limited mobility and eight $(29.6 \%)$ were bedridden. The underlying chronic conditions reported for VRE-positive patients, both in-patients and out-patients, included end-stage renal

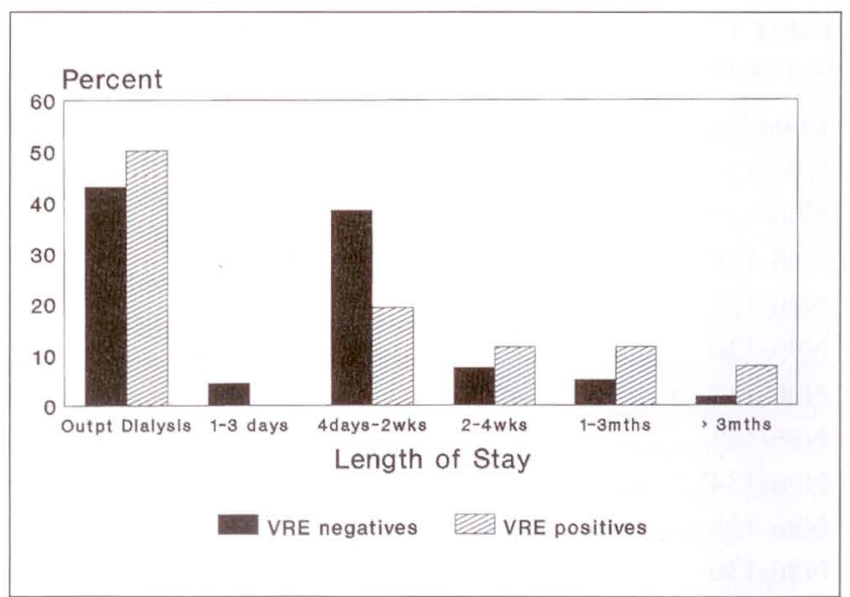

Figure 2) Length of stay. mths Months; Outpt Out-patient; VRE negatives Vancomycin-resistant enterococci (VRE) negative patients; VRE positives VRE-positive patients; wks weeks

disease, endocrine disorders (diabetes), central nervous system disorders (ie, dementia, stroke and peripheral neuropathy), gastrointestinal disorders (ie, cholangitis, duodenal diverticulitis, diverticular disease, ileostomy, partial bowel resection, stronglyloides with melena) and other disorders (primarily hypertension, coronary heart disease and chronic obstructive pulmonary disease).

Twenty (77\%) of the VRE-positive patients had surgery within the past year, with three cases having two separate surgeries within the past year and one case having three surgeries within the past year. Twelve (57\%) of these surgeries were intra-abdominal. Of those reported having surgery within the last year, $14(52 \%)$ had their surgery in the past three months, five $(19 \%)$ in the prior four to six months and eight $(30 \%)$ in the past seven to 12 months. Of the 14 VRE cases with an invasive procedure other than surgery, $11(78.6 \%)$ had a central line, five (35.7\%) had gastric, jejunal or nasogastric tubes, and one $(7.1 \%)$ had an endotracheal intubation tube.

The mean number of times the VRE-positive in-patients had been admitted to the hospital in the past year was four (range one to 15). The average number of in-patient days the VRE-positive patients experienced in the past 30 days was 13 (range zero to 30). One VRE-positive dialysis out-patient identified in Alberta had previously been hospitalized in London, United Kingdom. Only six other VRE-positive patients had reported being hospitalized in an institution other than the one in which they were tested - all six of these patients had been admitted to hospitals in metropolitan Toronto. Of the 24 VRE-positive dialysis patients, only three had been dialysed at an institution other than the present one within the past year. All out-patients had previously been admitted to a hospital an average of four times in the past year.

The fecal elimination pattern reported in VRE-positive patients included fecal incontinence (2), diarrhea (3) and normal fecal elimination (21). One VRE-positive patient also had urinary incontinence. Of the $11(42 \%)$ VRE-positive patients tested for $C$ difficile toxin, five (45\%) were positive. 


\section{DISCUSSION}

The CNISP 1996 point prevalence study identified 26 VREpositive patients. The patients were treated in two of the 26 participating hospitals. One hospital was identified as an endemic hospital because of an outbreak of VRE which occurred in the hemodialysis unit. This hospital reported 23 of the VRE-positive patients. The results of the 1996 VRE point prevalence surveillance project showed a prevalence of $0.1 \%$, in nonendemic hospitals, 3.7\% in endemic hospitals and $5.3 \%$ in endemic patient groups within endemic hospitals. No other patient-specific or laboratory-based national surveillance for VRE exists in Canada.

The endemic hospital had an outbreak of VRE in its renal dialysis population three months before this study (23). Over a four-month period, 41 patients were found to be colonized and one infected with vancomycin-resistant $E$ faecium. Of the 41 patients, 39 (95\%) were receiving dialysis, and two patients were contacts of dialysis patients. A case-control analysis revealed cases were significantly more likely to have had prolonged hospital stays, received two or more antibiotics, had diarrhea and/or fecal incontinence and greater than three hospital admissions in the previous year, all of which have been described previously. All the clinical isolates, except one, from this outbreak were vanB E faecium of a single clonotype.

The optimal approach with respect to the use of isolation precautions for patients colonized with VRE remains to be determined, but factors related to the likelihood of transmission (level of hygiene, continence, degree of illness) and the health care setting in which the patient is placed should be taken into consideration. Twenty-five of the VRE-positive patients identified in this survey reported being hospitalized or having received out-patient services from the endemic hospital. Biweekly to monthly follow-up of this cohort plus followup on additional patients in this population over a one-year period revealed that two-thirds of the affected individuals either intermittently or persistently carry VRE in their stools. Using an isolation protocol that reintegrated VRE-positive patients who were well, continent of stool and capable of good hygiene, only four new VRE colonized patients have been identified in the past year from over 400 screened cultures including all urine isolates of enterococci, all $C$ difficile stools, all sterile site isolates of enterococci and multiple ward-based screening surveys. Two of the four isolates were identified from patients transferred from facilities outside of the endemic hospital. The other two were identified following hospitalization in the endemic hospital; only one was epidemiologically linked to the original cohort of colonized patients. The use of isolation precautions based on individual risk assessment and reintegration of colonized VRE patients, who were otherwise well, has been effective at limiting the spread of VRE to other patients in this institution.

The one case in Alberta received dialysis for one month in London, United Kingdom before arriving in Canada. On entry to the dialysis program in Alberta in December 1995, this patient was screened and found to be colonized with VRE. This patient has since been receiving out-patient dialysis at the Alberta hospital and has been under a strict infection control protocol during treatments. The patient has remained colonized, and subsequent regular screening of all other patients receiving dialysis has been performed regularly within this hospital. To date, the isolation precautions implemented by this hospital have been effective in preventing the spread to any other patients.

Based in part on the results of this survey, LCDC, in collaboration with a subgroup of $\mathrm{CHEC}$, have developed guidelines for preventing the spread of VRE in Canada (24). These guidelines include a discussion of the microbiology, antibiotic resistance and susceptibility testing of enterococci, epidemiology and risk factors for VRE, and infection control activities to limit transmission.

In the CNISP point prevalence survey, one major PFGE type predominated (type A) in the E faecium van B isolates, suggesting that these isolates were epidemiologically linked. It is interesting to note that not all type A strains possessed the genes to confer resistance to tetracycline or doxycycline suggesting that the resistance may have been transferred during the course of the outbreak.

Several factors have been identified in other studies as risk factors for acquiring VRE. These factors include having $C$ difficile toxin in stools, geographical clustering of cases, intraabdominal surgery, multidrug therapy, duration of antibiotic use, prior exposure to vancomycin, duration of hospital stay, prior hospitalization, severe underlying disease or immunosuppression and prior nosocomial infections (15,25-27). In this survey, no statistically significant differences were seen between VRE-positive and -negative patients with respect to age, antibiotic use at the time of specimen collection and the number of antibiotics. Because information about antibiotic use and the number of antibiotics the patient received was collected only 'at specimen collection', antibiotic use before or after the point of specimen collection cannot be addressed in this study. None of the VRE-positive patients was on vancomycin. Because the endemic hospital had implemented restricted vancomycin usage throughout the hospital before this study, the fact that none of the VRE-positive patients in this study was on vancomycin likely reflects this restricted usage protocol. Similar to the findings of other studies, the VRE-positive patients identified in this survey were more likely to have been dialysis patients and to have a prolonged hospitalization before specimen collection. This may be attributed to the recent outbreak within the endemic hospital's renal dialysis population.

The VRE-positive patients identified in this point prevalence surveillance had long lengths of stay in hospital (mean 58 days), frequent hospital admissions (mean four per year) and severe admitting diagnoses and chronic underlying diseases. Many of the VRE-positive patients had multiple surgeries, a high rate for $C$ difficile toxin and a high prevalence of invasive procedures. This supports previous studies reporting that VRE is occurring in the highly impaired patient population.

Several limitations of these observations must be taken into account. It was not possible in this survey to distinguish whether VRE in the dialysis patients was a result of their higher risk or because an outbreak had occurred previously in this 
population. The latter, however, is much more likely because existent carriage of VRE has been well documented in this population. Scant data on carrier status and the identification of incidence cases limited our ability to identify potential modes of transmission and frequency of spread. Only those defined as high risk patients were included and therefore the prevalence of VRE outside these groups is not known.

\section{THE FUTURE}

Since the 1996 CNISP VRE point prevalence survey was conducted, outbreaks and occurrences of VRE have been re- ported in six other provinces. CNISP, therefore, plans to repeat a point prevalence survey in the first half of 1997. In addition, a case control study will be conducted in order to further define those at risk for VRE in Canadian health care institutions. After the 1997 point prevalence survey the data collected will be used by CNISP to determine prevalence, trends and the effects of vancomycin use control efforts. With strict infection control procedures, regular screening regimens and appropriate antimicrobial stewardship, the control of VRE within Canadian institutions may be successfully managed.

\section{REFERENCES}

1. Centre for Disease Control and Prevention. Addressing emerging infectious disease threats: A prevention strategy for the United States. MMWR 1994:43:1-18.

2. Centre for Disease Control and Prevention. Nosocomial enterococci resistant to vancomycin - United States, 1989-1993. MMWR 1993:42:597-9.

3. Schaberg DR, Culver DH, Gaynes RP. Major trends in the microbial etiology of nosocomial infections. JAMA 1991;91:72S-5S.

4. Zervos MJ, Terpenning MS, Schaberg DR, Therasse PM, Medendorp SV, Kauffman CA. High-level aminoglycosideresistant enterococci: Colonization of nursing home and acute care hospital patients. Arch Intern Med 1987;147:1591-4.

5. Wells CL, Juni BA, Cameron SB, et al. Stool carriage, clinical isolation, and mortality during an outbreak of vancomycin -resistant enterococci in hospitalized medical and/or surgical patient. Clin Infect Dis 1995;21:45-50.

6. Boyle JF, Soumakis AR, Herrington DG, Gianarkis DG, Thurberg BE, Painter BF. Epidemiologic analysis and genotypic characterization of a nosocomial outbreak of vancomycin-resistant enterococci. J Clin Microbiol 1993;31:1280-5.

7. Centre for Disease Control and Prevention. Statewide surveillance for antibiotic-resistant bacteria - New Jersey, 1992-1994. MMWR 1995;14:504-6.

8. Centre for Disease Control and Prevention. Assessment of testing for the completeness of reporting of vancomycin-resistant enterococci - Connecticut, 1994. MMWR 1996;45:289-91.

9. Centre for Disease Control and Prevention. Nosocomial enterococci resistant to vancomycin - United States, 1989-1993. MMWR 1993:42:597-9.

10. Communicable Disease Report Weekly. Vancomycin resistant enterococci in hospitals in the United Kingdom. Com Dis Rep Wkly 1995;5:281-4.

11. Sanyal D, Williams AJ, Johnson AP, George RC. The emergence of vancomycin resistance in renal dialysis. J Hosp Infect $1993 ; 24: 167-73$.

12. Rhinehart E, Smith NE, Wennersten C, et al. Rapid dissemination of beta-lactamase-producing aminoglycoside-resistant Enterococcus faecalis among patients and staff on an infant-toddler surgical ward. N Engl J Med 1990;323:1814-8.

13. Rubin LG, Tucci V, Cercenado E, Eliopoulos G, Isenber HD. Vancomycin-resistant Enterococcus faecium in hospitalized children. Infect Control Hosp Epidemiol 1992;13:700-5.

14. Coudron PE, Mayhall GC, Facklam RR, et al. Streptococcus faecium outbreak in a neonatal intensive care unit. J Clin Microbiol 1984;20:1044-8.

15. Boyle JF, Soumakis SA, Rendo A, et al. Epidemiologic analysis and genotypic characterization of a nosocomial outbreak of vancomycin-resistant enterococci. J Clin Microbiol 1993:31:1280-5.

16. Noble WC, Virani Z, Cree RGA. Co-transfer of vancomycin and other resistance genes from Enterococcus faecalis NCTC 12201 to Staphylococcus aureus. FEMS Micro Letters 1992;93:195-8.

17. Ofner ME, Conly J, Kureishi A, et al. Vancomycin resistant enterococcus (VRE) surveillance in Canada: The beginning of a new era. Infect Control Hosp Epidemiol 1996;17(Suppl 2):P20. (Abst)

18. Willey BM, Kreiswirth BN, Simor AE, et al. Detection of vancomycin resistance in Enterococcus species. J Clin Microbiol 1992;30:1621-4.

19. National Committee for Clinical Laboratory Standards. Methods for dilution of antimicrobial susceptibility tests for bacteria that grow anaerobically, 3rd edn [Publication M7-A3]. Villanova: National Committee for Clinical Laboratory Standards, 1995.

20. Dutka-Malen S, Evers S, Courvalin P. Detection of glycopeptide resistance genotypes and identification to the species level of clinically relevant enterococci by PCR. J Clin Microbial 1995;33:24-7.

21. Murray BE, Singh KV, Heath JD, Sharma BR, Weinstock GM. Comparison of genomic DNAs of different enterococcal isolates using restriction endonucleases with infrequent recognition sites. J Clin Microbiol 1990;28:2059-63.

22. Tenover FC, Arbeit RD, Goering RV, et al. Interpreting chromosomal DNA restriction patterns produced by pulse-field gel electrophoresis: Criteria for bacterial strain typing. J Clin Microbiol 1995;33:2233-9.

23. Lior L, Litt M, Hockin J, et al. Vancomycin-resistant enterococci on a renal ward in an Ontario hospital. Can Com Dis Rep 1996:22:125-8.

24. Health Canada. Guidelines for Preventing the Spread of Vancomycin-resistant Enterococci in Canada. VRE Working Group, Canadian Hospital Epidemiology Committee. Ottawa: Department of National Health and Welfare. (in press)

25. Karanfil LV, Murphy M, Josephson A, et al. A cluster of vancomycin-resistant Enterococcus faecium in an intensive care unit. Infect Control Hosp Epidemiol 1992;13:195-200.

26. Hospital Infection Control Practices Advisory Committee (HICPAC). Recommendations for preventing the spread of vancomycin resistance. Infect Control Hosp Epidemiol 1995;16:105-13.

27. Gordts B, Van Landuyt H, Ieven M, VanDamme P, Goossens H. Vancomycin-resistant enterococci colonizing the intestinal tracts of hospitalized patients. J Clin Microbiol 1995;33:2842-6. 


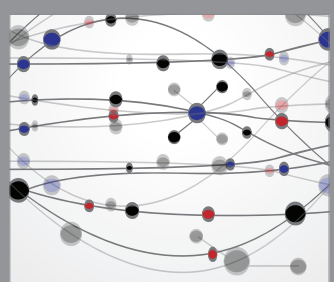

The Scientific World Journal
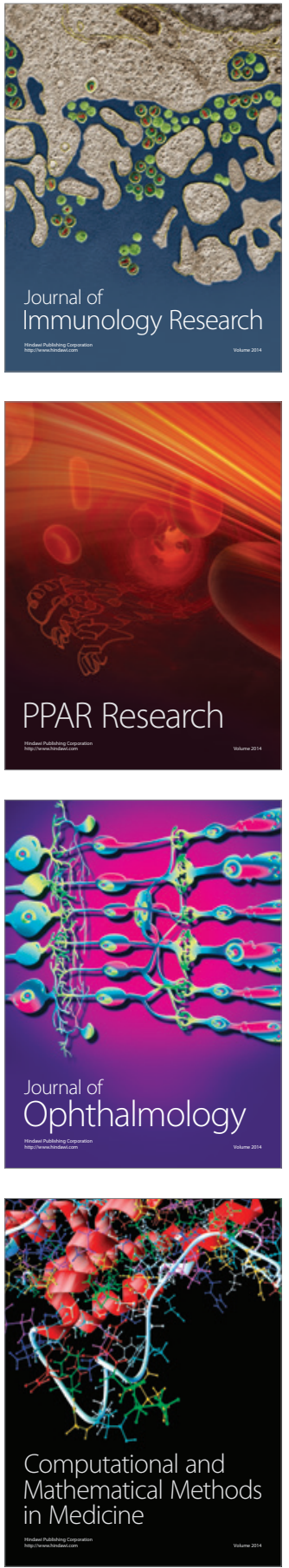

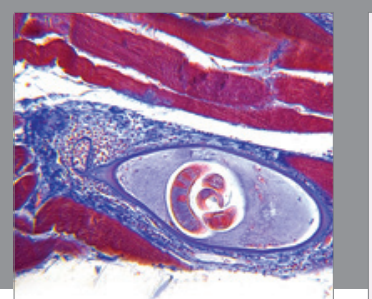

Gastroenterology Research and Practice

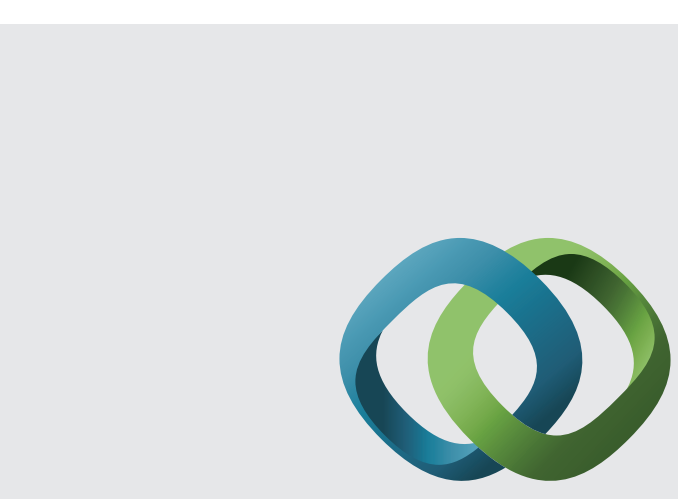

\section{Hindawi}

Submit your manuscripts at

http://www.hindawi.com
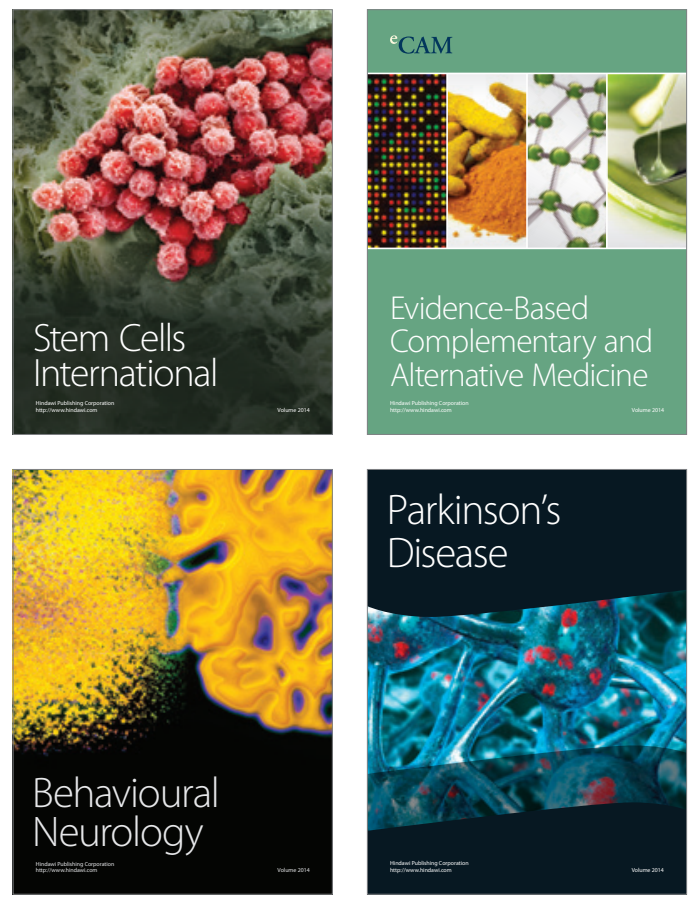
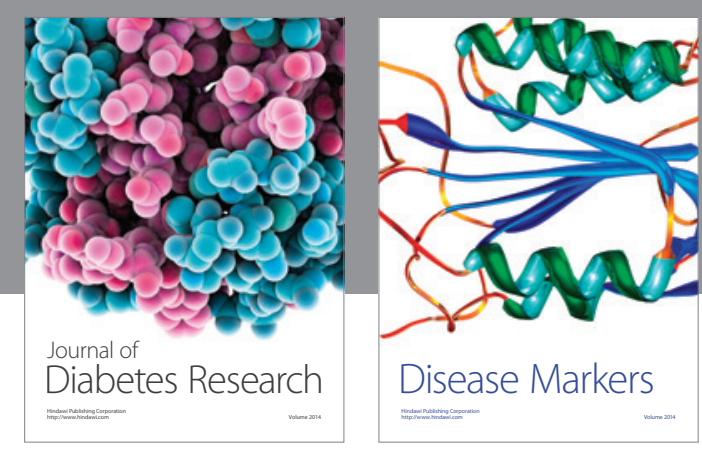

Disease Markers
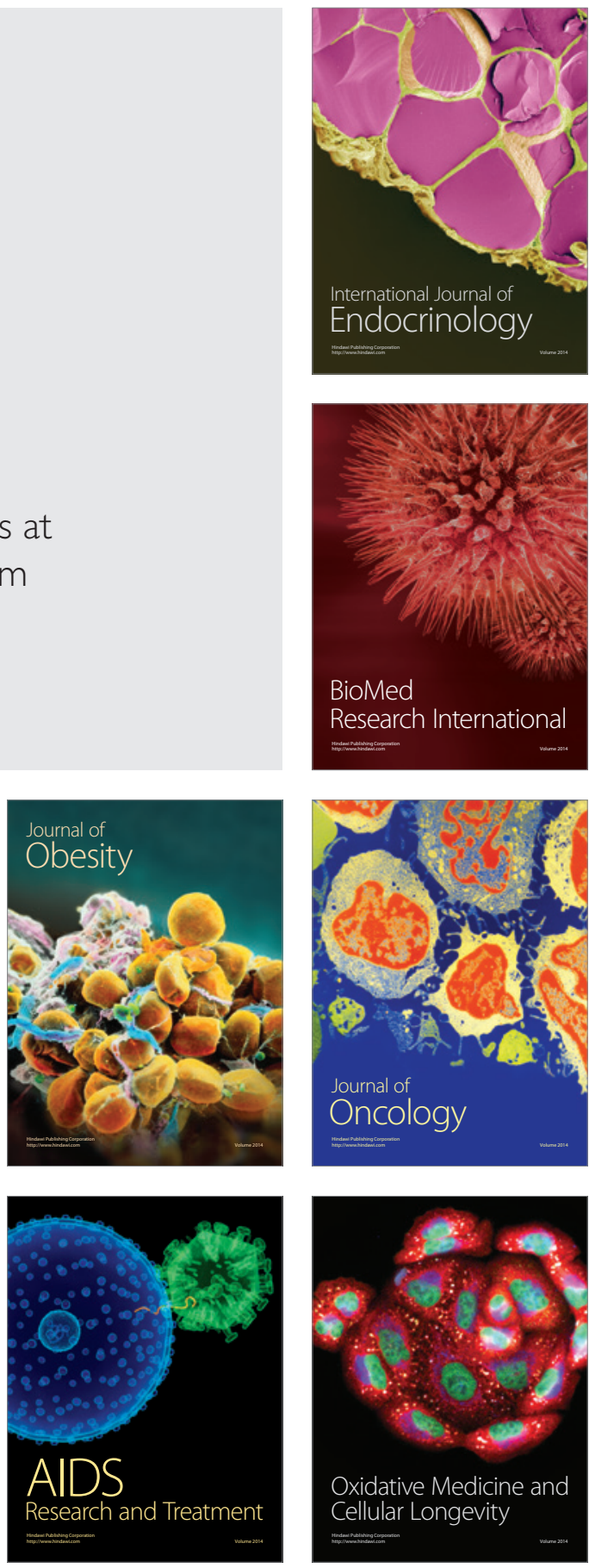